\section{Measurement of glucose concentrations in rats: differences between glucose meter and plasma laboratory results}

Dear Sir,

Experiments in rodents, especially rats and mice, serve as models for the investigation of physiological background and pathological findings in humans. Standards for laboratory values and procedures are necessary for comparison and reproducibility of experiments and results. For studies concerned with diabetes mellitus the most important standard is the determination of reliable glucose values in these animals. Such standards are not available on an up-to-date basis. Only some articles on laboratory animals dating back to the $1970 \mathrm{~s}$ and communications by providers of laboratory animals try to cover this topic [1-4]. We therefore did 265 measurements of blood glucose in normal and Streptozotocin-diabetic Lewis rats $(n=45)$ in the fasting and postprandial state in ranges between 2.7 and $27.7 \mathrm{mmol} / \mathrm{l}$. Measurements were determined from blood samples drawn from tail snips of Lewis rats. After spinning the ice-cooled whole blood samples for 2 minutes at $2000 \mathrm{rpm}$ the plasma samples were measured with a Beckman 2 Analyser (glucose oxidase method; Beckman, Palo Alto, Calif., USA). Samples prepared with or without sodium fluoride, which is known to prevent glycolysis, did not show different values. Fasting plasma values in normal rats were 5.6 plus or minus $0.1 \mathrm{mmol} / 1$, postprandial values were 6.0 plus or minus $0.2 \mathrm{mmol} / \mathrm{l}$. These results resemble those from the literature. To additionally address the question of differences between whole blood and plasma samples we made all measurements with the Beckman 2 Analyser and a portable glucose meter (One Touch II, Johnson and Johnson, Milpitas, Calif., USA), which is fast and easy to handle. Measurements with the One Touch II meter were done and thereafter the same samples were transferred for measurement with the Beckman 2 Analyser. The devices $(n=3)$ and the analyser were calibrated with code strips and standard solutions. The One Touch II meter was chosen after proving reliable in studies comparing new glucose meters with the glucose oxidase method $[5,6]$. In these studies, where human whole blood and plasma had been used, clinically relevant differences in accuracy and precision were measured. In our rodent study however, we tried to establish recommendations for laboratory standards. We found out that the following conversion factors from whole blood to plasma had to be used: a factor of 1.6 for blood glucose measurements from $2.7-5.5 \mathrm{mmol} / \mathrm{l}(n=112)$ and $5.5-11.1 \mathrm{mmol} / \mathrm{l}(n=62)$, a factor of 1.5 for values from $11.1-16.6 \mathrm{mmol} / \mathrm{l}(n=44)$, and a factor of 1.3 for values greater than $16.6 \mathrm{mmol} / \mathrm{l}(n=47)$. Dif-

Corresponding author: Dr.R. Weitgasser, 2nd Department of Medicine, Salzburg General Hospital, Muellner Hauptstrasse 48, A-5020 Salzburg, Austria ferences to measurements in humans could, in part, be due to higher haematocrit values in rats, $43-52 \%$ vs $36-50 \%$ in humans $[1-4,7]$. Especially in low blood glucose ranges this difference is enhanced by the fact that whole blood measured by the reflectometer contains $81 \%$ water whereas plasma which is used for glucose measurements with the Beckman 2 Analyser contains $93 \%$ water [8]. In summary the application of different conversion factors in accordance to various ranges of whole blood glucose measurements seems necessary. Extrapolation of these conversion factors for use with other blood glucose meters seems possible but comparative data in rodents are still lacking. These findings indicate that in rats, blood glucose concentrations measured with a blood glucose meter are substantially lower than plasma concentrations measured with the "gold standard" glucose oxidase method. These differences should be more widely appreciated by those working with rodent models of diabetes.

Yours sincerely,

R.Weitgasser, A. M.Davalli, G. C.Weir

\section{References}

1. Burns KF, de Lannoy CW (1966) Compendium of normal blood values of laboratory animals, with indication of variations. Toxicol Appl Pharmacol 8: 429-437

2. Burns KF, Timmons EH, Poiley SM (1971) Serum chemistry and hematological values for axenic (germfree) and environmentally associated inbred rats. Lab Animal Sci 21: 415-419

3. Ringler DH, Dabich L (1979) Hematology and clinical biochemistry. In: Baker HJ, Lindsey JR, Weisbroth SH (eds) The laboratory rat, vol I. Academic Press, New York, pp 105-120

4. Charles River Laboratories (1984) Hematology and clinical chemistry values for Charles River Fischer-344 rats$\mathrm{CDF}$ as a function of sex and age. CRL Technical Bulletin 3: $1-5$

5. Weitgasser R, Straberger A, Schnöll F, Sailer S (1994) Clinical evaluation of the blood glucose meters Accutrend, Companion 2, Glucometer 3 and One Touch II as compared with the reference glucose oxidase method. Wien Klin Wochenschr 106/23: 738-741

6. Brunner GA, Ellmerer M, Sendlhofer G et al. (1998) Validation of home blood glucose meters with respect to clinical and analytical approaches. Diabetes Care 21: 585-590

7. Williams WJ, Nelson DA, Morris MW (1990) Examination of the blood. In: Williams WJ, Beutler A, Erslev AJ, Lichtman MA (eds) Hematology, 4th edn. McGraw-Hill, New York, p 9

8. Quan AH, Cogan MG (1993) Body fluid compartments and water balance. In: Seldin DW, Giebisch G (eds) Clinical disturbances of water metabolism. Raven Press, New York, pp 1-9 